\title{
Holographic dynamics of unstable branes in AdS
}

\author{
Marija Zamaklar, Kasper Peeters \\ MPI/AEI für Gravitationsphysik, Am Mühlenberg 1, 14476 Golm, Germany \\ Available online 18 November 2004 \\ Presented by Guy Laval
}

\begin{abstract}
The gauge theory dual to the decay of an unstable D-particle in AdS is analysed in terms of coherent states. We discuss in detail how to count the number of particles in the decay product. We find, in agreement with the analysis in flat space, that the emission amplitude is suppressed as the mass of the radiated particles increases. To cite this article: M. Zamaklar, K. Peeters, C. R. Physique 5 (2004).

() 2004 Académie des sciences. Published by Elsevier SAS. All rights reserved.

\section{Résumé}

Dynamique holographique de branes instables dans AdS. La théorie de jauge duale à la désintégration de D-particules instables dans AdS est analysée en termes d'état cohérents. Nous discutons en détails le comptage du nombre de particule dans le produit de désintégration. Nous trouvons, en accord avec l'analyse en espace plat, que le taux d'émission est supprimé quand la masse de la particule augmente. Pour citer cet article: M. Zamaklar, K. Peeters, C. R. Physique 5 (2004).

๑) 2004 Académie des sciences. Published by Elsevier SAS. All rights reserved.

Keywords: Gauge theory; Unstable branes

Mots-clés : Théorie de jauge; Branes unstables
\end{abstract}

\section{Introduction}

Tachyon condensation provides an interesting arena in which we can improve our understanding of string theory in a dynamical set-up. While the condensation of closed string tachyons, and the associated decay of spacetimes, is still hampered by conceptual and technical problems, a lot of progress has recently been made in understanding the dynamics of open string tachyons. Most of the analysis was performed directly using boundary conformal field theory in flat space, initiated by Sen's construction of the boundary states for decaying D-branes [1], or by using the $c=1$ matrix model for the description of the decay of D-branes in $1+1$ dimensional string theory [2]. In the present work we study the problem of decaying branes in the set-up of the 'standard' AdS/CFT correspondence.

As was argued by Harvey et al. [3], unstable D-branes in string-theory are equivalents of 'sphalerons': they are unstable solutions located at a saddle point of the potential in string field theory configuration space, at the top of a non-contractible loop [4]. In the context of the AdS/CFT conjecture, this correspondence between unstable D-branes and sphalerons in gauge theory is in fact even more direct. By analysing the kinematical properties of these two systems, it has been argued by Drukker

E-mail addresses: marija.zamaklar@aei.mpg.de (M. Zamaklar), kasper.peeters@aei.mpg.de (K. Peeters). 
et al. [5] that the unstable D-particles of string theory are in precise correspondence with known sphaleron solutions of the dual gauge theory.

We will study dynamical properties of this correspondence. On the gravity side we start with the results of Lambert et al. [6] for the spectrum of decaying D-branes in flat space. To compare these results to those which we will obtain in gauge theory, we 'embed' the flat-space results in the AdS space. A priori, there is no reason to expect that the flat space results of the decay should be valid for branes in an AdS background. However, since the D-particles in question are fully localised in the bulk space, one expects that the flat space results should carry over, at least when the radius of the AdS is large.

There are two properties of the spectrum of the decaying brane that we want to compare with the dual gauge theory calculation. The first property of the spectrum is constrained by the symmetries of the system, and concerns emission amplitudes for the states on the leading Regge trajectory. By slightly refining the calculation of [6] we find [7] that all emission amplitudes for these states are zero. The same result is separately recovered on the gauge theory side by evaluating the number operator for the corresponding dual composite operators.

More important is a second property of the spectrum, observed in [6], which reflects genuine dynamical features of the decay. There is strong evidence [1,6] that the open strings decay fully into closed string states, i.e. that there is no open string remnant left after the decay. This conclusion is also supported by the matrix model calculations of [2]. As shown in [6], the emission amplitudes are exponentially suppressed with the level of the emitted string, at least for high levels (however, due to the exponential growth of the available states, most of the energy of the brane gets transferred into a high-density cloud of very massive closed string states).

In the remainder of this article we focus on two issues in the dual gauge theory on the boundary. The first issue is the construction of the time-dependent gauge theory solution which is the analogue of Sen's time-dependent boundary state in boundary conformal field theory. The second issue is how, given this time dependent solution, one can reproduce the two properties of the spectrum of the decaying particle mentioned above.

\section{D-particle $\leftrightarrow$ sphaleron correspondence: statics}

Before addressing the dynamical properties of the correspondence, let us first briefly revise its basic static properties [5]. Gauge theory sphalerons [4] are static solutions of the equations of motion, associated to saddle points whose existence is guaranteed by the existence of a non-contractible loop in (compact) configuration space. Whereas the sphaleron solution on $\mathbb{R}^{4}$ in Yang-Mills-Higgs theory, found by Klinkhamer and Manton [8], is very complicated and not known analytically, the situation is much simpler on $S^{3} \times \mathbb{R}$ in pure Yang-Mills theory. To construct the sphaleronic gauge configuration in the $S U(2)$ gauge theory, one starts from the instanton solution on $\mathbb{R}^{4}$,

$$
A_{\mu}=f(r)\left(\partial_{\mu} U\right) U^{\dagger}, \quad U=\frac{x^{\mu} \sigma_{\mu}}{r}, \quad r^{2}=x_{0}^{2}+x_{i}^{2},
$$

where $f=r^{2} /\left(r^{2}+a^{2}\right)$. This function interpolates between two pure gauge configurations (i.e. the two vacua) $f(r=0)=0$ and $f(r=\infty)=1$. When $f(r)=1 / 2$, the system is at the top of the potential barrier, see Fig. 1 . By taking $f=1 / 2$ everywhere one gets a singular solution to the equation of motion on $R^{4}$, which is the so-called 'meron'. The $f=1 / 2$ solution is, however, also a solution on $S^{3} \times \mathbb{R}$, since this manifold can be conformally mapped to $\mathbb{R}^{4}$ and Yang-Mills theory in four dimensions is conformally invariant. The solution obtained in this way is the Euclidean version of the 'sphaleron', and is non-singular. The
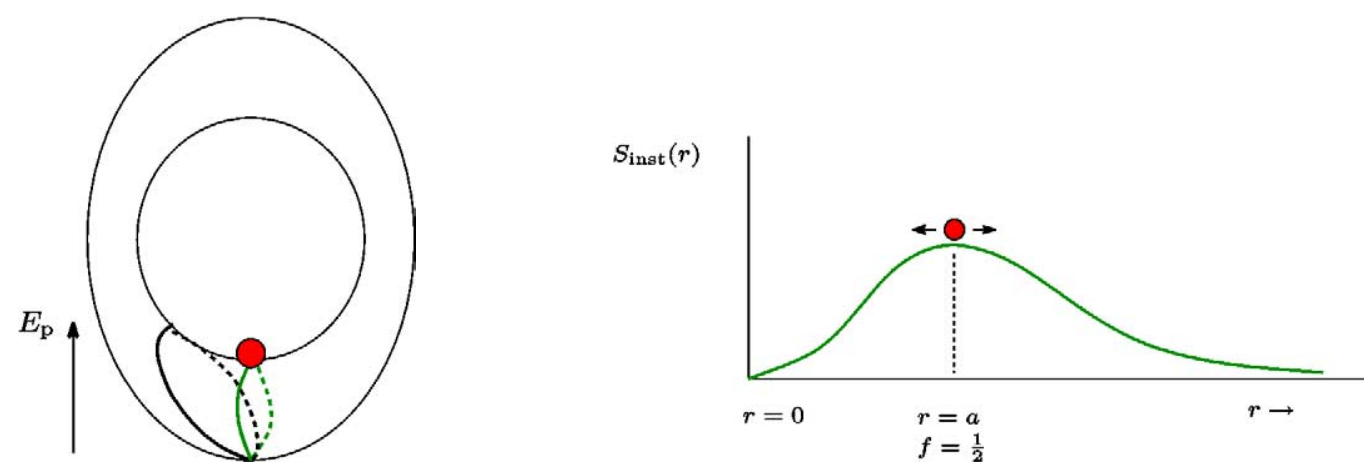

Fig. 1. The picture on the left shows in a schematic way the existence of a non-contractible loop in configuration space, as well as the presence of the sphaleron (large dot) at the saddle point. The picture on the right shows the action density $S(r)$ of the instanton in the Euclidean theory, together with the special configuration at $r=a$ which is used to construct the sphaleronic particle in the Lorentzian theory. 
Lorentzian version is the same, since the time component of the potential of the sphaleron is zero. The solution is completely time-independent and has infinite action, corresponding to a sphaleronic particle which is sitting at the top of the potential.

As far as a generalisation of the previous construction to $S U(N)$ gauge theory is concerned, the general sphaleron configuration is not known. However, an interesting special configuration has been given in [5]. It is obtained by replacing the Pauli matrices in (1) with Clifford algebra generators according to

$$
\sigma_{\mu} \rightarrow \gamma_{\mu}=\left(\begin{array}{cccc}
\sigma_{\mu} & 0 & \cdots & 0 \\
0 & \sigma_{\mu} & \cdots & 0 \\
\vdots & \vdots & \ddots & 0 \\
0 & 0 & \cdots & \sigma_{\mu}
\end{array}\right)
$$

The mass of this sphaleronic particle is $k$ times the mass of the $S U(2)$ particle (where $k$ is the number of sigma matrices in (2) and $2 k<N$ ). It was also shown that the number unstable modes is increased from one (for $S U(2))$ to $k^{2}$.

It has been argued by Drukker et al. [5] that the (non-supersymmetric) sphaleronic saddle points in the gauge theory are preserved as the 't Hooft coupling is increased, despite the fact that the precise form of the potential receives quantum corrections. The main reason for this is that these sphaleronic saddle points are linked to the underlying non-contractible loops in configuration space. Furthermore, they are linked to the (supersymmetric) instanton configuration which is present both at strong and weak coupling. Thus the sphaleronic particle in the Yang-Mills theory on $S^{3} \times \mathbb{R}$ has, at weak coupling, been conjectured to be dual to the unstable D-particle in the AdS.

A number of arguments has been given [5] in support of this correspondence. Firstly, both D-particles and sphaleronic particles are static with respect to the global AdS time. Secondly, since the D-particle is located at the origin of the AdS space (in global coordinates), it is 'projected' in a homogeneous fashion to the boundary, in agreement with the fact that the sphaleronic particle is homogeneously spread over the $S^{3}$. Thirdly, the D-particle in the bulk is a source for the gravitational and dilaton field (while it does not source the RR forms), which is in agreement with the (non)vanishing expectation values of the dual gauge operators. Finally, in the case of the more general sphaleron (2), the number of unstable modes on both sides agrees.

\section{D-particle $\leftrightarrow$ sphaleron correspondence: dynamics}

To study the dynamics of the decaying D-particles from the gauge theory perspective let us, as a first step, construct the time dependent gauge configuration describing the sphaleron decay. We restrict to the decay modes which preserve spherical symmetry by making the following ansatz,

$$
A=f(t) \Sigma^{i} \sigma_{i}
$$

where $\Sigma^{i}$ are the three left-invariant one-forms. To deduce what is the unknown function $f(t)$ we plug the ansatz into the action and derive the action for this function. The value of the action for our ansatz is

$$
S=-\frac{1}{4 g_{\mathrm{YM}}^{2}} \int \mathrm{d} t \mathrm{~d} \Omega F_{\mu \nu} F^{\mu \nu}=\frac{24 \operatorname{vol}\left(S^{3}\right)}{4 g_{\mathrm{YM}}^{2}} \int \frac{\mathrm{d} t}{R}\left(\frac{R^{2}}{2} \dot{f}^{2}-2 f^{2}(1-f)^{2}\right),
$$

where $\operatorname{vol}\left(S^{3}\right) \equiv 2 \pi^{2}$ denotes the volume of the unit sphere and $R$ is the radius of $S^{3}$. The equation of motion for the function $f$ is

$$
R^{2} \ddot{f}+4 f(1-f)(1-2 f)=0 .
$$

When integrated once, this equation yields a conserved quantity, namely the energy (i.e. the component $T_{00}=48 \operatorname{vol}\left(S^{3}\right) E$ )

$$
E=R^{2} \dot{f}^{2}+4 f^{2}(1-f)^{2}
$$

which is simplest to integrate analytically for $E=1 / 4$. There are two solutions, corresponding to the fact that the sphaleron can roll down on either side of the potential, to the vacua with Chern-Simons number one and zero respectively. The final result reads (see Fig. 2)

$$
f_{ \pm}(t)=\frac{1}{2}\left(\frac{ \pm \sqrt{2}}{\cosh \left(\frac{\sqrt{2}}{R}\left(t-t_{0}\right)\right)}+1\right) .
$$

This solution describes a configuration that starts from the potential maximum at $t=-\infty$ (with zero velocity and acceleration), rolls down the hill and up the other side, where it arrives at $t=t_{0}{ }^{1}$

\footnotetext{
${ }^{1}$ After we had derived this solution, we learned that it has been obtained before [9], albeit in a different context.
} 


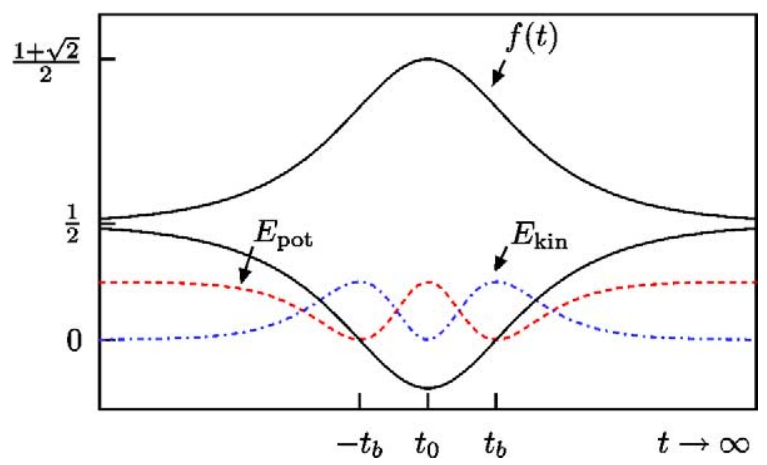

Fig. 2. The functions $f_{ \pm}(t)$ of the decaying sphaleron on $S^{3}$ as given in (7), together with the kinetic and potential energy (with normalisation as given in (6) and $R=1$ ).

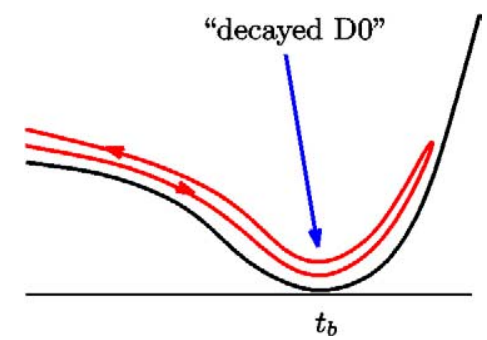

Fig. 3. The evolution of the sphaleron. As it rolls down, it reaches a point where all potential energy has been converted to kinetic energy. This is what we will call the 'decayed D-particle', despite the fact that the decay products will eventually come back as fine-tuned radiation to 're-build' the D-particle.

The periodicity of the whole process is natural from the AdS perspective. Since AdS effectively acts as a box, the cloud of outgoing radiation is refocused to the origin of the space, where it arrives as fine-tuned radiation and 're-builds' the D-particle. In this sense the D-particle never decays, since there is no real dissipation of the energy in the system. However, in the limit of large AdS radius, our flat-space intuition should (at least approximately) hold. A natural point in time, which should be associated to the decayed brane, is the point where the sphaleron has rolled down to the bottom of the potential, i.e. when all potential energy has been converted to kinetic energy (see Fig. 3).

Near the bottom the solution is

$$
A_{\mu}=\tilde{f}(t) U^{\dagger}\left(\partial_{\mu} U\right), \quad \tilde{f}=f-1
$$

with $\tilde{f} \approx 0$, which means that the derivative part of the field strength, rather than the non-linear (commutator) part, is dominant. The solution becomes a solution of the free Yang-Mills equations of motion on $S^{3} \times \mathbb{R}$ (written in the radiation gauge: $A_{0}=$ $\left.\nabla_{i} A^{i}=0\right)$,

$$
\left(-\partial_{t}^{2}+\frac{1}{R^{2}}\left(\nabla_{S^{3}}^{2}-2\right)\right) A_{i}^{\text {lin. }}=0 .
$$

Indeed, one can easily see that as $t \rightarrow t_{\text {bottom }}$ the solution (8) with $f$ given by (7) is very well approximated by the following solution of the linearised equation of motion (9):

$$
A_{i}^{\text {lin. }}=-\frac{1}{4} \sin \left(\frac{2\left(t-t_{\text {bottom }}\right)}{R}\right) U^{\dagger}\left(\partial_{i} U\right) .
$$

Hence near the bottom of the valley, one can think about the Yang-Mills configuration as dual to a coherent state of noninteracting closed string states which are the product of the D-particle decay. Our goal will then be to determine the numbers of various (gravity) 'particles' in this final coherent state. What we precisely mean by this will be explained in the next section. Let us first construct this coherent state. 
The fact that our solution abelianises near the bottom of the potential allows us to apply the standard machinery to write down the coherent state. By expanding the classical, free Yang-Mills gauge potential in terms of spherical vector harmonics, one can read off the amplitudes for different modes, and write a coherent state as

$$
|c\rangle=\mathcal{C} \exp \left(g_{\mathrm{YM}}^{-2} \sum_{J, M, y} \operatorname{Tr}\left(A_{J M y} \hat{a}_{J M y}^{\dagger}\right)\right)|0\rangle,
$$

where $A_{J M y}$ are the coefficients appearing in the Fourier decomposition of the classical sphaleron configuration and the normalisation factor $\mathcal{C}$ is chosen such that $|c\rangle$ is of unit norm.

For this to be a legitimate state in the Hilbert space, one has to make sure that it satisfies all constraints. It is easy to see that creation operators in (11) lead to physical excitations in the free theory. However, once $g_{Y M}$ is turned on, Gauss' law implies that only singlets can be excited. This means that all non-singlet states in (11) have to be projected out. In practice, however, we will neither write this projector nor construct the projected state explicitly. This is because our calculations always involve projections of the coherent state onto states which themselves are color singlets. Therefore the singlet projection is imposed implicitly throughout.

\section{Particles in the AdS/CFT correspondence}

In the AdS/CFT correspondence we have a relation between string states in the bulk and operators in the boundary. These operators are, via the operator-state mapping, interpreted to create 'particles' in the bulk theory at a particular point on the boundary. That is, one needs to solve for the wave equation of the dual field in the bulk in the presence of a delta source inserted at the boundary. This means that the states created in the bulk are not eigen-momentum states, an attribute which one usually associates to the notion of a particle in field theories. However, since the AdS/CFT correspondence is formulated in position space rather than momentum space, these definitions are natural in this context. On the other hand, our string calculation in [7] is a flat space calculation, and for us it will be more natural to use the standard notion of particles in the bulk as angular momentum eigenstates. Therefore, we will first have to construct boundary operators that are dual to bulk angular momentum eigenstates.

The operator-state correspondence is usually discussed in the context of radial quantisation of conformal field theories (see, e.g., [10] for a discussion in a four-dimensional context). One first Wick rotates $\mathbb{R} \times \mathbb{R}^{3}$ to the Euclidean regime and then performs a conformal transformation such that the origin of $\mathbb{R}^{4}$ corresponds to $t=-\infty$ in the original frame. Operators inserted at the origin are then in one-to-one correspondence with states in the Hilbert space. The entire procedure can, however, be formulated without doing the conformal rescaling, which is more natural in our setup since, as we have discussed before, the gauge field configuration on $\mathbb{R} \times S^{3}$ is non-singular while the one on $\mathbb{R}^{4}$ is singular.

The state corresponding to an operator with conformal weight $w$ is obtained by multiplying with the appropriate exponential of Euclidean time and taking the limit $\tau \rightarrow-\infty$ (keeping only the regular part):

$$
\left|\hat{O}_{\text {weight- } w}^{(m)}\right\rangle=\lim _{\tau \rightarrow-\infty}\left\{e^{-w \tau} \hat{O}_{\text {weight- } w}^{(m)}(\tau)\right\}|0\rangle \equiv \hat{O}_{\text {weight }-w}^{\dagger(m)}|0\rangle .
$$

The last expression shows the shorthand notation that we will use in order not to clutter expressions unnecessarily. The hermitian conjugate of an operator is given by

$$
(\hat{O}(\tau))^{\dagger}=\hat{O}^{\dagger}(-\tau)
$$

This procedure mimics the operator-state mapping on $\mathbb{R}^{4}$ but avoids technical problems related to solutions which become singular after the conformal transformation.

The operators which we use in (12) are independent of the angular coordinates on the sphere, i.e. they are obtained from the position dependent operators as follows

$$
\hat{O}_{w}^{(m)}(\tau)=K_{w}^{(m)} \int_{S^{3}} \mathrm{~d} \Omega \hat{O}_{w}^{\mu_{1} \cdots \mu_{s}}\left(\tau, \phi_{i}\right) Y_{\mu_{1} \cdots \mu_{s}}^{(m)}\left(\phi_{i}\right) .
$$

Here $Y^{(m)}$ denote the lowest lying tensor spherical harmonics for a given spin $s$. The index $m$ labels the degeneracy of such harmonics. The normalisation constants $K_{w}^{(m)}$ are chosen such that the states constructed using (12) are of unit norm. Note that the multiplication with the time dependent exponent in (12) selects out composite operators of the required conformal dimension, but when one expresses these operators in terms of elementary creation and annihilation operators, one explicitly sees that different operators $\hat{O}$ are not orthogonal. It is only after the integration (14) that one obtains a set of orthogonal states.

There are many subtleties related to the fact that operators $\hat{O}$ are composite operators rather than elementary gauge operator. Firstly, the multi-particle states cannot simply be obtained by acting repeatedly with the $\hat{O}^{\dagger}$ operators on the vacuum. States 
generated in this way are not orthogonal, not even in the $N \rightarrow \infty$ limit when the number of operators becomes large as well. Starting from the naive states $\left(\hat{O}^{\dagger}\right)^{n}|0\rangle$ one has to subtract terms in order to achieve orthogonality. For the same reason, there is no simple number operator which can be used to count the number of composite excitations in a given state. It is true that

$$
\left[\hat{O}, \hat{O}^{\dagger}\right]=1+\mathcal{O}\left(N^{-2}\right)
$$

and one might expect that this leads to a well-defined number operator $\hat{O}^{\dagger} \hat{O}$. However, the coefficients that multiply the $1 / N^{2}$ corrections in (15) are operators, not c-numbers. As a consequence, the strength of the $1 / N^{2}$ corrections depends on the state in which the number operator is evaluated,

$$
\left\langle n\left|\hat{O}^{\dagger} \hat{O}\right| n\right\rangle=n+\sum_{i} \frac{c_{i}(n)}{N^{2 i}} .
$$

The numbers $c_{i}(n)$ can become arbitrarily large when $n \rightarrow \infty$. Since the coherent state contains such highly excited states, the operator $\hat{O}^{\dagger} \hat{O}$ cannot be used as a number operator, not even in the $N \rightarrow \infty$ limit. $^{2}$ We will encounter an explicit manifestation of these problems in the next section, when we start counting particles in the coherent state, and then on a concrete example we will illustrate how one can deal with them.

Let us end this section with a comment on alternatives to the coherent state (11). From the point of view of the dual string theory, it might seem more natural to construct a coherent state using the composite operators $\hat{O}_{J}^{\dagger}$ in the exponent, rather than the elementary ones $\hat{a}^{\dagger}$. After all, the $\hat{O}_{J}$ correspond to elementary string excitations. However, a state of the form

$$
|\tilde{c}\rangle=\tilde{\mathcal{C}} \exp \left(\sum_{i} O_{i}^{\text {class. }} \hat{O}_{i}^{\dagger}\right)|0\rangle
$$

is not a coherent state in the standard sense since the expectation value of an operator in this coherent state does not equal the classical value of that operator,

$$
\left\langle\tilde{c}\left|\hat{O}_{i}\right| \tilde{c}\right\rangle \neq O_{i}^{\text {class. }}
$$

not even up to $1 / N$ corrections. The reason for this is essentially given in Eq. (16), with $|n\rangle$ now being given by $|n\rangle=\left(\hat{O}_{i}^{\dagger}\right)^{n}|0\rangle$. This is our prime motivation to use (11) as the sphaleron coherent state.

\section{Particle counting}

Starting from the coherent state (11) we now want to extract information from it about particle numbers in the decay product. By particle counting, we mean counting of the states constructed in the previous section.

Due to the problems explained around (15), one cannot use the 'standard' number operator $\hat{O}^{\dagger} \hat{O}$. Instead we will simply decompose the coherent state on the basis of multi-particle states. Subsequently we will, using these probabilities, calculate the average energies and particle numbers. The probability of finding a multi-particle state consisting of $p_{1}$ particles of type $O_{J_{1}}$, $p_{2}$ particles of type $O_{J_{2}}$ etc., is given by

$$
\mathcal{P}\left(p_{1} ; p_{2} ; \ldots ; p_{M}\right):=\frac{\left|\left\langle\left(\hat{O}_{J_{1}}\right)^{p_{1}} \ldots\left(\hat{O}_{J_{M}}\right)^{p_{M}} \mid c\right\rangle\right|^{2}}{\left\langle\left(\hat{O}_{J_{1}}\right) p_{1} \ldots\left(\hat{O}_{J_{M}}\right) p_{M} \mid\left(\hat{O}_{J_{1}}\right) p_{1} \cdots\left(\hat{O}_{J_{M}}\right) p_{M}\right\rangle\langle c \mid c\rangle} .
$$

For this to work it is of course crucial that the basis of multi-particle states is constructed to be orthogonal. By definition, the average number of particles of the type $\hat{O}_{J_{i}}$ present in the coherent state is now given by

$$
N\left(J_{i}\right):=\sum_{p_{1}=0}^{\infty} \cdots \sum_{p_{M}=0}^{\infty} p_{i} \mathcal{P}\left(p_{1} ; p_{2} ; \ldots ; p_{M}\right) .
$$

The energy stored in these particles, as measured with respect to the global time in the bulk, is given by the conformal dimension of the corresponding operators. Therefore, the total energy is given by the expression

$$
E\left(J_{i}\right):=\sum_{p_{1}=0}^{\infty} \cdots \sum_{p_{M}=0}^{\infty} \Delta_{J_{i}} p_{i} \mathcal{P}\left(p_{1} ; p_{2} ; \ldots ; p_{M}\right),
$$

\footnotetext{
${ }^{2}$ An proper number operator for composite particles, which produces the exact occupation number rather than an expression which is only correct up to $N^{-2}$ corrections, has been constructed by [11]. However, their operator is very complicated and difficult to handle in practice. We prefer to follow a different route here.
} 
where $\Delta_{J_{i}}$ is the conformal dimension of the operator $\hat{O}_{J_{i}}$. For a generic operator, the calculation of the numerators in (19) reduces to evaluating the classical expression of the (abelianised) operator using the positive frequency part of the decayed solution. Hence, by considering only the numerators in (19) we can deduce which particles are absent from the decay spectrum. In particular one can easily deduce that expectation values of the operators dual to the graviton, NS-NS two form and all twist two operators are zero. ${ }^{3}$ By slightly refining the calculation of [6] we have found that all emission amplitudes for these states are zero in string theory as well [7]. The absence of the gravitational radiation is not surprising, since the decay is spherically symmetric. We also believe that absence of the other states is dictated by some underlying symmetry arguments.

Thus, to explore the genuine symmetry aspects of the decay we need to concentrate on the states for which (19) does not vanish. The main technical problem arises when evaluating the denominators of (19). To illustrate this, let us consider a 'simplified' model, based on a non-abelian scalar field. This model exhibits all of the technical subtleties associated to the determination of the decay products. The crucial ingredients of the vector coherent state, namely that it is constructed from the lowest-lying spherical harmonics and that it depends non-perturbatively on the coupling constant, are preserved by this toy model. It, however, avoids the inessential technical complications associated to the evaluation of tensor spherical harmonics in the numerators of (19).

The coherent state for a given classical configuration in this non-abelian scalar theory is given by

$$
|c\rangle=\mathcal{C} \exp \left(\frac{1}{g_{\mathrm{YM}}^{2}} \operatorname{Tr}\left(a \hat{a}^{\dagger}\right)\right)|0\rangle, \quad \mathcal{C}=\exp \left(-\frac{1}{g_{\mathrm{YM}}^{2}} \operatorname{Tr}\left(a^{\dagger} a\right)\right) .
$$

This mimics the construction (11). The unit normalised (at leading order in $1 / N$ expansion), single-trace operators which create particles in the out vacuum are

$$
\hat{O}_{J}^{\dagger}=\frac{1}{\sqrt{J\left(g_{\mathrm{YM}}^{2} N\right)^{J}}} \operatorname{Tr}\left(\left(\hat{a}^{\dagger}\right)^{J}\right) .
$$

These operators are coordinate independent operators, obtained using a procedure similar to (14).

With the above normalisation of the operator, the numerators and hence probabilities in (19) depend on the Yang-Mills coupling in a non-perturbative fashion,

$$
\left|\left\langle 0\left|\left(\hat{O}_{J}\right)^{p}\right| c\right\rangle\right|^{2}=\mathcal{C}^{2}\left|\frac{\operatorname{Tr}\left(\left(a^{+}\right)^{J}\right)}{\sqrt{J\left(g_{\mathrm{YM}}^{2} N\right)^{J}}}\right|^{2 p} \equiv \frac{\mathcal{C}^{2}}{J^{p}}\left(\frac{\eta_{J}^{2}}{\lambda^{J}}\right)^{p}
$$

(where the last equality defines $\eta_{J}$; note that it is of the order $N$ for the configuration (2) and generically scales as the number of D-particles). This reflects the fact that our original sphaleron configuration is a non-perturbative solution of the equations of motion. Note also that the only way in which the coupling $\lambda$ appears in (20) and (21) is through the combination $\eta_{J}^{2} / \lambda^{J}$.

The complicated part of the calculation of the average particle numbers and energies is the computation of the norms for the states with an arbitrary number of particles. The norm of the state with $p$ identical particles can be written as

$$
\begin{aligned}
\left\langle\left(\hat{O}_{J}\right)^{p}\left(\hat{O}_{J}^{\dagger}\right)^{p}\right\rangle= & p !\left\langle\left(\hat{O}_{J}\right)\left(\hat{O}_{J}^{\dagger}\right)\right\rangle^{p}+\left(\begin{array}{c}
p \\
2
\end{array}\right)^{2}\left\langle\left(\hat{O}_{J}\right)^{2}\left(\hat{O}_{J}^{\dagger}\right)^{2}\right\rangle_{\text {connected }}(p-2) !\left\langle\left(\hat{O}_{J}\right)\left(\hat{O}_{J}^{\dagger}\right)\right\rangle^{(p-2)} \\
& +\left(\begin{array}{c}
p \\
3
\end{array}\right)^{2}\left\langle\hat{O}_{J}^{3} \hat{O}_{J}^{\dagger 3}\right\rangle_{\text {connected }}(p-3) !\left|\hat{O}_{J} \hat{O}_{J}^{\dagger}\right\rangle^{(p-3)} \\
& +\left(\begin{array}{c}
p \\
2
\end{array}\right)^{2}\left(\begin{array}{c}
p-2 \\
2
\end{array}\right)^{2}\left\langle\left(\hat{O}_{J}\right)^{2}\left(\hat{O}_{J}^{\dagger}\right)^{2}\right\rangle_{\text {connected }}^{2} \frac{(p-4) !}{2 !}\left\langle\hat{O}_{J} \hat{O}_{J}^{\dagger}\right\rangle^{(p-4)}+\cdots
\end{aligned}
$$

The first term is at a leading order independent of $1 / N$, the second is suppressed as $1 / N^{2}$, the last two terms both scale as $1 / N^{4}$, and so on. A similar but more complicated expansion can be written for states involving more than one type of particle.

Naively, one might expect that in the large- $N$ limit, all but the leading term $p$ ! in this expansion can be omitted. However, this would produce an exponential dependence on the expectation values for the operators $\hat{O}_{J}$ in formula (20). Since the arguments of the exponent (24) increase with conformal dimension $J$, one would conclude that the number of particles produced during the decay increases with the mass of the particle. It is easy to see that this kind of truncation of (25) does not make sense in the case of the non-perturbative coherent state (22), as it would actually produce probabilities (19) which are larger than one.

\footnotetext{
${ }^{3}$ Note that the expression which vanishes is the energy momentum tensor evaluated on the positive frequency part of the solution: $\left|\left\langle 0\left|\hat{T}_{\mu \nu}\right| c\right\rangle\right|^{2}=\left|T_{\mu \nu}\left(A_{\text {coherent }}^{+}\right)\right|^{2}=0$. On the other hand, the classical expression for the energy momentum tensor of the full configuration is non-zero: $T_{\mu \nu}\left(A^{+}+A^{-}\right) \neq 0$.
} 
The point is that since the numerator (24) is very large, the maximal probabilities are attained for large values $p^{\text {max }}$ of $p$. Moreover, $p^{\max }$ grows with $N$, hence in the large- $N$ limit the sub-leading terms in (25) become more and more relevant, and are actually comparable to the leading term.

In trying to estimate how fast the norms (25) have to grow with $p$, one can see that even an exponential growth of the norms, say as $p ! \gamma^{p}(\gamma=\mathrm{const})$, does not lead to reasonable results. Namely, if we consider the expression $\sum_{p} \mathcal{P}(J, p)$, which has to be smaller than one, and assume exponential growth of norms, we would find that this sum behaves as

$$
\sum_{p=0}^{\infty} \mathcal{P}(J, p)=\mathcal{C}^{2} \sum_{p=0}^{\infty} \frac{1}{p !}\left(\frac{\eta_{J}^{2}}{\lambda^{J} \gamma}\right)^{p}=\exp \left(\frac{\eta_{J}^{2}}{\lambda^{J} \gamma}\right) \exp \left(-\frac{N}{\lambda} \operatorname{Tr}\left(a^{\dagger} a\right)\right) .
$$

Hence we see that even when $N \rightarrow \infty$ (while keeping $\lambda$ arbitrary but smaller than one) the result will always be larger than 1 for some value of $J$. Since the calculation of the average number of particles requires a summation over all $J$, we conclude that we cannot assume this behavior of the norms. ${ }^{4}$

The situation which we face here is similar in spirit to the double-scaling BMN limit. As observed in [12] and [13], in the limit $N \sim J^{2} \rightarrow \infty$ correlators in general receive contributions from non-planar graphs of all genera. In this case, a new expansion parameter $J^{2} / N$ appears. In our case, $N \rightarrow \infty$ as well, but now the additional parameter which becomes large is the value of the $p_{i}$ for which the sum (21) has its maximum term. It would be interesting to understand whether our system also exhibits a double-scaling limit in which some ratio of powers of $p$ and $N$ is kept fixed.

\section{Calculation of norms and numerical results}

In order to determine the correct values of the norms of the states, it is useful to write the norms of multi-particle states in terms of correlators of a complex matrix model,

$$
\begin{aligned}
\langle 0| & {\left[\left(\hat{O}_{J_{1}}\right)^{p_{1}} \cdots\left(\hat{O}_{J_{n}}\right)^{p_{n}}\right]\left[\left(\hat{O}_{J_{n}}^{\dagger}\right)^{p_{n}} \cdots\left(\hat{O}_{J_{1}}^{\dagger}\right)^{p_{1}}\right]|0\rangle } \\
& =\int \mathrm{d} A \mathrm{~d} \bar{A}\left[\left(O_{J_{1}}\right)^{p_{1}} \cdots\left(O_{J_{n}}\right)^{p_{n}}\right]\left[\left(O_{J_{n}}^{\dagger}\right)^{p_{n}} \cdots\left(O_{J_{1}}^{\dagger}\right)^{p_{1}}\right] \exp \left(-\operatorname{Tr}\left(A^{\dagger} A\right)\right) .
\end{aligned}
$$

The measure used here is simply a separate integral over the real and imaginary parts of the complex matrix $A$, normalised to give unit result when all $p_{i}$ in the expression above are zero,

$$
\int \mathrm{d} A \mathrm{~d} \bar{A}=\pi^{-N} \prod_{a, b=1}^{N} \mathrm{~d}\left(\operatorname{Re} A_{a b}\right) \mathrm{d}\left(\operatorname{Im} A_{a b}\right) .
$$

This approach has been used by $[14,12]$ in order to compute several special cases of (28) analytically. It is still an open problem to extend those exact results to the entire class of correlators, in particular to general situations for which $p_{i}>2$. Because we will need these very general correlators, we have decided to use an alternative approach, in which the integral is evaluated using Monte-Carlo methods. This provides us with a technically straightforward way to extract the norms for arbitrary operator insertions, even for very large $p_{i}$. Our results will, for this reason, of course be restricted to a fixed value for $N$ and computer resources put a practical limit on the maximum value that can be handled (we will take $N=4$ ). Nevertheless, we will see that interesting results can be obtained this way.

In the $U(4)$ theory there are only two operators which create physical states (using only the creation operator for the lowestlying spherical harmonics). These are $\operatorname{Tr}\left(\left(a^{\dagger}\right)^{2}\right)$ and $\operatorname{Tr}\left(\left(a^{\dagger}\right)^{4}\right) .^{5}$ The proper linear combinations of these operators are

$$
\hat{O}_{2}^{\dagger}=\operatorname{Tr}\left(a^{\dagger} a^{\dagger}\right), \quad \hat{O}_{4}^{\dagger}=\operatorname{Tr}\left(a^{\dagger} a^{\dagger} a^{\dagger} a^{\dagger}\right)-\frac{2 N^{2}+1}{N\left(N^{2}+2\right)} \operatorname{Tr}\left(a^{\dagger} a^{\dagger}\right) \operatorname{Tr}\left(a^{\dagger} a^{\dagger}\right) .
$$

\footnotetext{
${ }^{4}$ Note that if we would have had a perturbative coherent state instead of a non-perturbative one, the classical expectation values $a$ in (22) would be of the form $a=g_{\mathrm{YM}} \eta$, with $\eta$ a number independent of the coupling constant. Hence formula (26) would be replaced with

$$
\sum_{p=0}^{\infty} \mathcal{P}(J, p)=\mathcal{C}^{2} \sum_{p=0}^{\infty} \frac{1}{p !}\left(\frac{\eta_{J}^{2}}{N^{J} \gamma}\right)^{p}=\exp \left(\frac{\eta_{J}^{2}}{N^{J} \gamma}\right) \exp \left(-\operatorname{Tr}\left(a^{\dagger} a\right)\right)
$$

We now see that a truncation to the first term in (25) (i.e. setting $\gamma=1$ ) produces reasonable results for the probabilities (19).

5 The restriction to the zero-mode of the scalar field is motivated by the full sphaleron solution of the earlier sections, which only turns on the lowest spherical vector harmonics. Naturally, in the full $U(4)$ there are also operators of the form $\operatorname{Tr}\left(D_{\mu} \phi D_{\nu} \phi\right)$. However, in the oscillator picture these are turned on by the oscillators that create the higher spherical tensorial harmonics.
} 


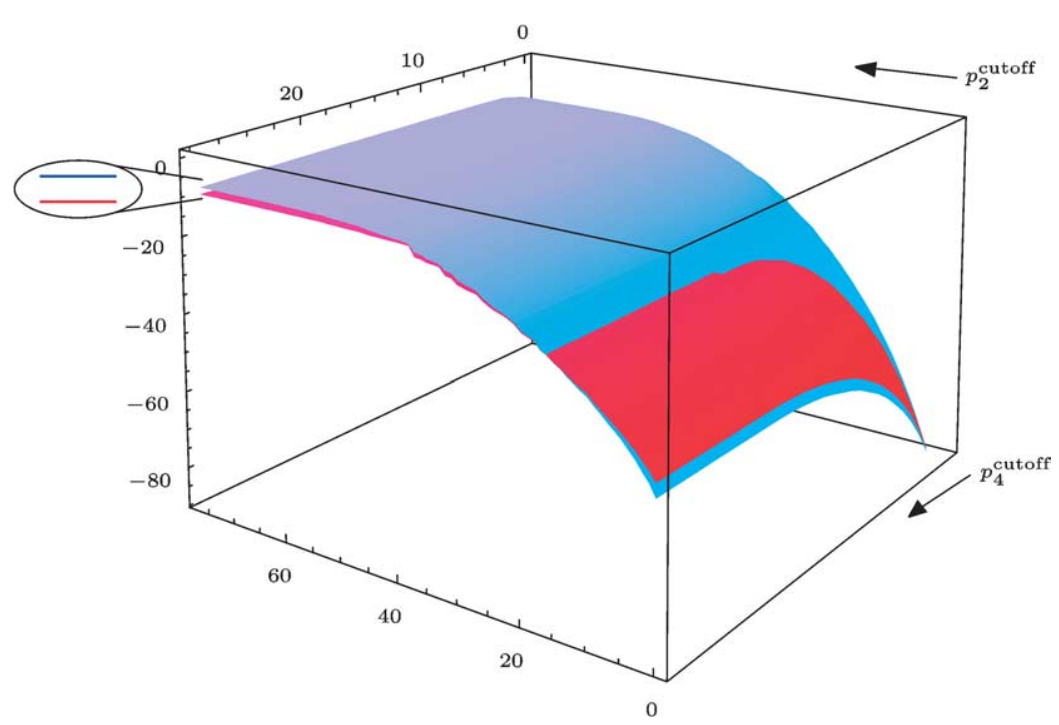

Fig. 4. Successive approximations to the logarithm of the total energy radiated in the $J=2$ particles (light, blue surface) and $J=4$ particles (dark, red surface). The $x$ and $y$ axes label the maximum value of $p_{2}$ and $p_{4}$ in the sum (32). The values asymptote to the full result in the upper left corner of the graph. While the present plot shows energies, qualitatively similar plots are obtained for the particle numbers.

These lead to $\left\langle\hat{O}_{4} \mid \hat{O}_{2} \hat{O}_{2}\right\rangle=0$. Multi-particle states will generically not be orthogonal, but in our case this turns out to be far less important than the $1 / N^{2}$ corrections to the norms. We will for simplicity also use a classical configuration for which

$$
\frac{\eta_{4}}{N}=\left(\frac{\eta_{2}}{N}\right)^{2}=\frac{\eta}{N}
$$

where the $\eta_{J}$ are defined in (24). Closer inspection of the coherent state of the sphaleron given in (11) shows that the expectation values of, e.g., the $\operatorname{Tr}\left(F_{m n} F^{m n}\right)$ and $\operatorname{Tr}\left(F_{m n} F^{m n} F_{r s} F^{r s}\right)$ states are similarly related.

The energy radiated into $O_{J=2}$ and $O_{J=4}$ particles can be computed using formula (21), summed over a suitably large range of values for $p_{2}$ and $p_{4}$. In our particular case, this formula reduces to

$$
E\left(J, p_{2}^{\text {cutoff }}, p_{4}^{\text {cutoff }}\right)=\sum_{p_{2}=0}^{p_{2}^{\text {cutoff }}} \sum_{p_{4}=0}^{p_{4}^{\text {cutoff }}}\left|\frac{\eta_{2}^{2}}{\lambda^{2}}\right|^{p_{2}}\left|\frac{\eta_{4}^{2}}{\lambda^{4}}\right|^{p_{4}} \frac{J p_{J}}{2^{p_{2} p_{4}}} \frac{\mathcal{C}^{2}}{\left\langle 0\left|\left(\hat{O}_{2}\right) p_{2}\left(\hat{O}_{4}\right)^{p_{4}}\left(\hat{O}_{4}^{\dagger}\right) p_{4}\left(\hat{O}_{2}^{\dagger}\right) p_{2}\right| 0\right\rangle\langle c \mid c\rangle}
$$

and the maximum values of $p_{2}$ and $p_{4}$ which are included in the sum should be taken sufficiently large as to include at least the maximum term in the sum. This requirement is indeed met in our numerical approach. We have computed the ratio of energies in the $J=2$ and $J=4$ particles using successive approximations of (32), for larger and larger $p_{2}^{\text {cutoff }}$ and $p_{4}^{\text {cutoff }}$, for a range of couplings. ${ }^{6}$ A typical example is plotted in Fig. 4. One clearly sees that the asymptotic value of the ratio $E(4) / E(2)$, given by the exponent of the asymptotic height difference between the two surfaces, is smaller than one. We therefore conclude that our calculation predicts that higher-energy states in the decay product are suppressed with respect to the lower-energy ones. This is in qualitative agreement with alternative calculations of this decay process [6].

It would be very interesting to extend our analysis to higher-rank gauge groups, perhaps by obtaining an analytic expression for the norms of the states. For $N>4$, there are more than two gauge singlet states, and it becomes possible to determine the suppression factor as a function of the energy in more detail. We leave this for future investigations.

\footnotetext{
${ }^{6}$ From the gravity point of view, in case of large $N$, the $p^{\text {cutoff }}$ should always be such that the total energy (i.e. conformal dimension) carried by this multi particle state is smaller than $N^{2}$ in order to neglect back reaction. In the case of small $N$, such as discussed here, constants of order one become relevant, and this rough estimate is no longer sufficient. For example, it turns out [7] that the maximal probability for the number of particles of type $\mathcal{O}_{2}$ is larger than $N^{2}$, but the total energy carried by these particles is still smaller than the energy of the brane (once constants of order one have been taken into account).
} 


\section{Summary and outlook}

We have presented the formalism to analyse the decay of unstable D-branes in the $\mathrm{AdS}_{5} \times S^{5}$ background by considering the dual gauge theory. Our results show qualitative agreement with previous work on D-particle decay, and our work provides a basis for further study of non-perturbative dynamical features of the correspondence.

A very relevant way of improving on our results would be to determine analytical expressions for the norms required in Section 6. This would allow one to extend the results obtained there to large values of $N$. Also, as we have explained, due to the non-perturbative nature of the initial sphaleron configuration, the computation of the decay product requires information from a regime in which both $N \rightarrow \infty$ as well as the number of particles $p \rightarrow \infty$. Knowing the norms of states analytically should allow us to understand this double limit. This perhaps may circumvent the need to calculate the norms of states exactly when calculating the energy distribution in the final state. Finally, it would be interesting to understand how quantum corrections can be incorporated into our formalism, in order to see how much they influence the qualitative characteristics of the decay product.

\section{Acknowledgements}

We thank Gleb Arutyunov, Rajesh Gopakumar, Justin David, Stefano Kovacs, Charlotte Kristjansen, Shiraz Minwalla, Jan Plefka and Ashoke Sen for discussions.

\section{References}

[1] A. Sen, Rolling tachyon, JHEP 0204 (2002) 048, hep-th/0203211.

[2] J. McGreevy, H. Verlinde, Strings from tachyons: the $c=1$ matrix reloaded, 2003, hep-th/0304224.

[3] J.A. Harvey, P. Horava, P. Kraus, D-sphalerons and the topology of string configuration space, JHEP 0003 (2000) 021; hep-th/0001143.

[4] N.S. Manton, Topology in the Weinberg-Salam theory, Phys. Rev. D 28 (1983) 2019.

[5] N. Drukker, D.J. Gross, N. Itzhaki, Sphalerons, merons and unstable branes in AdS, Phys. Rev. D 62 (2000) 086007; hep-th/0004131.

[6] N. Lambert, H. Liu, J. Maldacena, Closed strings from decaying D-branes, 2003, hep-th/0303139.

[7] K. Peeters, M. Zamaklar, AdS/CFT description of D-particle decay, 2004, hep-th/0405125.

[8] F.R. Klinkhamer, N.S. Manton, A saddle point solution in the Weinberg-Salam theory, Phys. Rev. D 30 (1984) 2212.

[9] G.W. Gibbons, A.R. Steif, Yang-Mills cosmologies and collapsing gravitational sphalerons, Phys. Lett. B 320 (1994) 245-252; hepth/9311098.

[10] S. Fubini, A.J. Hanson, R. Jackiw, New approach to field theory, Phys. Rev. D 7 (1973) 1732-1760.

[11] W.E. Brittin, A.Y. Sakakura, Number operators for composite particles in nonrelativistic many body theory, J. Math. Phys. 21 (1980) 2164-2169.

[12] C. Kristjansen, J. Plefka, G.W. Semenoff, M. Staudacher, A new double-scaling limit of $N=4$ super Yang-Mills theory and pp-wave strings, Nuclear Phys. B 643 (2002) 3-30; hep-th/0205033.

[13] N.R. Constable, D.Z. Freedman, M. Headrick, S. Minwalla, Operator mixing and the BMN correspondence, JHEP 0210 (2002) 068; hep-th/0209002.

[14] N. Beisert, C. Kristjansen, J. Plefka, G.W. Semenoff, M. Staudacher, BMN correlators and operator mixing in $N=4$ super Yang-Mills theory, Nuclear Phys. B 650 (2003) 125-161; hep-th/0208178. 